\title{
ON THE ASYMPTOTIC BEHAVIOR OF SOLUTIONS OF SECOND ORDER LINEAR DIFFERENTIAL EQUATIONS
}

WILLIAM F. TRENCH

In this paper we study the behavior of solutions of the differential equation

$$
u^{\prime \prime}=(f(t)+g(t)) u
$$

as $t \rightarrow \infty$. We assume that the general solution of

$$
z^{\prime \prime}=f(t) z
$$

is known, and prove the following theorem.

Theorem. Let $z_{1}$ and $z_{2}$ be independent solutions of (2), let $g$ be continuous in $0 \leqq t<\infty$, and assume that

$$
\int_{0}^{\infty}|g| y d t<\infty
$$

where

$$
y(t)=\max \left[\left|z_{1}(t)\right|^{2},\left|z_{2}(t)\right|^{2}\right] .
$$

Then, if $a$ and $b$ are arbitrary constants, there is a solution of (1) which can be written in the form

$$
u=\alpha(t) z_{1}+\beta(t) z_{2},
$$

with $\lim _{t \rightarrow \infty} \alpha(t)=a$ and $\lim _{t \rightarrow \infty} \beta(t)=b$.

In the proof of this theorem, we use a lemma due to Bellman $[1$, p. 35].

LEMMA. If $u, v \geqq 0$, if $c_{1}$ is a positive constant, and if

$$
u \leqq c_{1}+\int_{0}^{t} u v d t_{1}
$$

then

$$
u \leqq c_{1} \exp \left(\int_{0}^{t} v d t_{1}\right) .
$$

ProOF OF THE THEOREM. We write

Received by the editors October 19, 1961 and, in revised form, December 20, 1961. 


$$
u=A z_{1}+B z_{2}
$$

and require that

$$
A^{\prime} z_{1}+B^{\prime} z_{2}=0 .
$$

Differentiating (4) twice yields

$$
u^{\prime \prime}=A z_{1}^{\prime \prime}+B z_{2}^{\prime \prime}+A^{\prime} z_{1}^{\prime}+B^{\prime} z_{2}^{\prime} \text {. }
$$

From (2) and (4), the sum of the first two terms is $f(t) u$. Hence, from (1) and (4),

$$
A^{\prime} z_{1}^{\prime}+B^{\prime} z_{2}^{\prime}=g\left(A z_{1}+B z_{2}\right) .
$$

Equations (5) and (6) can be solved to yield

$$
\begin{aligned}
& A^{\prime}=\frac{g z_{2}}{W}\left(A z_{1}+B z_{2}\right), \\
& B^{\prime}=\frac{-g z_{1}}{W}\left(A z_{1}+B z_{2}\right),
\end{aligned}
$$

where $W=z_{1}^{\prime} z_{2}-z_{1} z_{2}^{\prime}=$ constant. Integrate (7) to obtain

$$
\begin{aligned}
& A(t)=A(0)+\frac{1}{W} \int_{0}^{t} g z_{2}\left(A z_{1}+B z_{2}\right) d x \\
& B(t)=B(0)-\frac{1}{W} \int_{0}^{t} g z_{1}\left(A z_{1}+B z_{2}\right) d x
\end{aligned}
$$

for $t \geqq 0$. From this it follows that

$$
\begin{aligned}
|A(t)|+|B(t)| \leqq & |A(0)|+|B(0)| \\
& +\frac{2}{|W|} \int_{0}^{t}|g| y(|A|+|B|) d x .
\end{aligned}
$$

From Bellman's lemma

$$
|A(t)|+|B(t)| \leqq(|A(0)|+|B(0)|) \exp \frac{2}{|W|} \int_{0}^{t}|g| y d x
$$

for positive $t$, and therefore, from (3), $A$ and $B$ are bounded. Now it follows that the integrals on the right sides of (8) and (9) are convergent at infinity, so that $\lim _{t \rightarrow \infty} A(t)$ and $\lim _{t \rightarrow \infty} B(t)$ exist and are finite.

Let $A_{1}(t)$ and $B_{1}(t)$, and $A_{2}(t)$ and $B_{2}(t)$ be the solutions of (7) such that $A_{1}(0)=1, B_{1}(0)=0, A_{2}(0)=0, B_{2}(0)=1$. Denote $\lim _{t \rightarrow \infty} A_{i}(t)=a_{i}$, and $\lim _{t \rightarrow \infty} B_{i}(t)=b_{i} \quad(i=1,2)$. We have shown above that these 
limits exist. The function $A_{1} B_{2}-A_{2} B_{1}$ is a constant (unity), since its derivative vanishes identically, as may be seen from (7). Hence $a_{1} b_{2}-a_{2} b_{1}=1$, and it is easy to verify that $\alpha(t)$ and $\beta(t)$, defined by

$$
\begin{aligned}
& \alpha(t)=\left(b_{2} a-a_{2} b\right) A_{1}(t)+\left(b a_{1}-a b_{1}\right) A_{2}(t), \\
& \beta(t)=\left(b_{2} a-a_{2} b\right) B_{1}(t)+\left(b a_{1}-a b_{1}\right) B_{2}(t),
\end{aligned}
$$

satisfy the requirements of the theorem.

This theorem contains a previously known result $[1$, p. 112] to the effect that, if every solution of (2) is bounded, and if $\int_{0}^{\infty}|g| d t<\infty$, then every solution of (1) is bounded. It also contains results of Fubini [2], who studied the special cases $f(t)=1$ and $f(t)=-1$.

For the special case $f(t) \equiv 0$, one can conclude from the present theorem that, if $\int_{0}^{\infty}|g| t^{2} d t<\infty$, then every solution of $u^{\prime \prime}=g(t) u$ can be written in the form $u=A(t)+B(t) t$, where $A$ and $B$ approach prescribed limits. This in turn contains a result of Sansone [3], demonstrated under the assumption that $\left.|g(t)|\left\langle k t^{-c}, k\right\rangle 0, c\right\rangle 3$.

Acknowledgment. I thank the referee for pointing out that the theorem could be stated in its present form, which is stronger than my original result, and for drawing my attention to the results of Fubini and Sansone.

\section{REFERENCES}

1. R. Bellman, Stability theory of differential equations, McGraw-Hill, New York, 1953.

2. G. Fubini, Studi asintotici per alcune equazioni differenziali, Rend. Reale Accad. Lincei 26 (1937), 253-259.

3. G. Sansone, Equazioni differenziali nel campo reala, 2nd ed., Nicola Zanichelli, Bologna, 1948.

The Radio Corporation of America 